\title{
Evidências de validade da Escala de Consciência Plena no Trabalho (ECPT)
}

\author{
André Luis Amorim Silva Filho \\ Maria Cristina Ferreria \\ Felipe Valentini \\ Universidade Salgado de Oliveira, RJ, Brasil
}

\begin{abstract}
Resumo
Foi objetivo do estudo reunir evidências de validade de estrutura interna e convergente da Escala de Consciência Plena no Trabalho. Participaram do estudo 383 trabalhadores, de ambos os sexos (65,7\% do sexo feminino), com idades variando entre 18 e 70 anos $(M=34,5 ; D P=11,7)$, que responderam à versão brasileira da escala e a instrumentos para avaliação de outros construtos. As análises fatoriais confirmatórias demonstraram a existência de sete itens concentrados em um único fator, com índice de consistência interna de 0,81 . Na validação convergente, a escala correlacionou-se positiva e moderadamente com o engajamento laboral, a saúde mental e os afetos positivos dirigidos ao trabalho, e negativa e moderadamente com o neuroticismo. A escala apresentou, portanto, evidências de validade de estrutura interna, de precisão e de validade convergente, o que recomenda seu uso futuro em pesquisas e diagnósticos organizacionais sobre a consciência plena no trabalho.
\end{abstract}

Palavras-chave: Análise fatorial; Validade estatística; Consciência; Trabalho; Saúde.

\section{Evidences of validity of the Mindfulness at Work Scale}

\begin{abstract}
The objective of the study was to gather evidences of internal structure and convergent validity the Mindfulness at Work Scale. Study participants were 383 workers of both sexes $(65.7 \%$ female), aged between 18 and 70 years $(M=34.5, S D=11.7)$, who answered the Brazilian version of the scale and instruments to assess other constructs. The factor analysis demonstrated the existence of seven items concentrated on a single factor, with internal consistency index of 0.81 . In convergent validity, the scale correlated positively and moderately with work engagement, mental health and positive affects directed to work, and negative and moderately with neuroticism. The scale had therefore evidence of internal structure of validity, reliability and convergent validity which recommends its use in future research and organizational diagnoses of mindfulness at work.
\end{abstract}

Keywords: Factor analysis; Statistical validity; Consciousness; Work; Health.

\section{Evidencias de validez de la Escala de Atención Plena en el Trabajo}

\section{Resumen}

El objetivo del estudio era reunir pruebas de validez de estructura interna y convergente de la escala de Atención Plena en el Trabajo. Participarón del estudio 383 trabajadores de ambos sexos (65,7\% mujeres), con edades comprendidas entre 18 y 70 años $(M=34,5, S D=11,7)$, que respondieron a la versión brasileña de la escala y instrumentos de evaluación de otros construtos. El análisis factorial demostró la existencia de siete ítens centrados en un solo factor, con el índice de consistencia interna de 0,81 . La escala se correlacionó positiva y moderadamente con la vinculación laboral, la salud mental y los afectos positivos, y negativa y moderadamente con el neuroticismo. Las evidencias de validez de la estructura interna, de precisión y de validez convergente recomiendan el uso de la escala en futuras investigaciones y diagnósticos de la atención plena en el trabajo.

Palabras clave: Análisis factorial; Validez estadística; Atención; Trabajo; Salud. 
A busca pela compreensão dos fatores que contribuem para o bem-estar e a felicidade das pessoas intensificou-se sobremaneira nas últimas décadas (Houben, Noortgate, \& Kuppens, 2015). No escopo desta discussão, encontra-se um fenômeno que vem sendo estudado principalmente nos âmbitos da psicologia clínica e da personalidade e que, apenas mais recentemente, passou a interessar também aos estudiosos da psicologia organizacional e do trabalho. Trata-se da consciência plena no trabalho, ou seja, o grau em que o trabalhador mostra-se plenamente consciente do que ocorre em seu ambiente laboral (Dane \& Brummel, 2013).

Os instrumentos destinados a avaliar tal construto são, porém, escassos. Dessa forma, para avaliar a consciência plena no trabalho ao nível individual, o único instrumento disponível, até o momento, é a Escala de Consciência Plena no Trabalho (ECPT), desenvolvida por Dane e Brummel (2013). No Brasil, no entanto, não há, até o momento, nenhuma escala construída e/ou adaptada com a finalidade de avaliar a consciência plena no trabalho, além de haver uma grande carência de investigações destinadas a elucidar as consequências adversas da falta de atenção no ambiente de trabalho e da realização automática e superficial das tarefas atribuídas aos trabalhadores, conforme levantamento realizado nas bases de dados Scielo e Pepsic, em março de 2017.

Justificam-se, assim, estudos que possam buscar evidências adicionais sobre a validade da Escala de Consciência Plena no Trabalho (ECPT) em amostras ainda não investigadas anteriormente, como é o caso, por exemplo, do contexto brasileiro. Tal investigação poderá contribuir para o diagnóstico futuro do grau em que o trabalhador demonstra plena consciência do que ocorre em seu ambiente laboral e para o desenvolvimento de pesquisas destinadas ao aprofundamento da rede nomológica que cerca o referido construto, especialmente no que diz respeito a suas relações com o bem-estar laboral e a qualidade de vida no trabalho, bem como para a adoção de práticas laborais que se mostrem mais saudáveis para os trabalhadores e eficazes para as organizações. Fundamentando-se nessas considerações, foi objetivo geral do presente estudo adaptar e reunir evidências iniciais de validade de estrutura interna e convergente dos escores da ECPT em uma amostra de trabalhadores brasileiros.

\section{A consciência plena e sua mensuração}

O conceito psicológico de consciência plena diz respeito à integração de respostas cognitivas e emocionais não avaliativas e não reativas (Kopelman,
Avi-Yonah, \& Varghese, 2012), destinadas a aumentar a qualidade da atenção, capacitando, assim, o indivíduo a responder de forma mentalmente habilidosa a situações adversas do dia a dia (Bishop et al., 2004). Ela envolve, portanto, a aceitação da realidade tal qual ela se apresenta, no instante em que acontece, e a adoção de ações de maneira plenamente conscientes, sem a emissão de juízos irrefletidos ou reações automáticas às demandas da vida (Kopelman et. al., 2012).

$\mathrm{O}$ fenômeno oposto à consciência plena denomina-se inconsciência plena (mindlessness, em língua inglesa) e define-se pela ausência dessa característica. Desse modo, quando a atenção de um indivíduo encontra-se dividida e envolvida com múltiplas tarefas, ou quando ele age de forma automática ou reativa, ele não está em sua consciência plena (Brown \& Ryan, 2003). Ao vivenciar a inconsciência plena, as pessoas tornam-se, portanto, ausentes, suas mentes vagueiam, a atenção dirige-se a preocupações do passado ou do futuro e seus pensamentos e emoções tendem a distraílas ou sobrecarregá-las. O estado de mindlessness é mais comumente observado entre as pessoas do que o de consciência plena (Marianetti \& Passmore, 2010).

As pesquisas têm demonstrado que pessoas submetidas a intervenções com base na consciência plena apresentam redução da depressão (KlaininYobas, Cho, \& Creedy, 2012) e do estresse percebido (Wolever et. al., 2012), bem como melhoras na qualidade do sono (Winbush, Gross, \& Kreitzer, 2007), na frequência cardíaca (Wolever et. al., 2012), no bemestar psicológico e na qualidade de vida (Nyklíček, \& Kuijpers, 2008). As escalas desenvolvidas com o propósito de mensurar a consciência plena ainda não são muitas, em função da introdução desse construto na literatura psicológica ainda ser relativamente recente. Ainda assim, os instrumentos já desenvolvidos com esse propósito exibem apoio teórico consistente e propriedades psicométricas promissoras.

De particular interesse para o presente trabalho é a Mindful Attention Awareness Scale (MAAS) (Brown \& Ryan, 2003). Na construção da escala, os autores partiram do pressuposto de que os indivíduos diferem em sua propensão de se mostrarem conscientes e de estarem atentos, razão pela qual a consciência pode ser considerada como um estado que varia de um polo totalmente consciente (mindfulness) a um polo que denota a ausência total de tal consciência (mindlessness).

Tomando por base tal definição, os autores da escala construíram 184 itens, que foram testados em sete diferentes amostras norte-americanas (que incluíram desde praticantes de meditação Zen a pessoas sem qualquer experiência meditativa), reunidas em 
cinco estudos distintos. Adotando procedimentos de análise fatorial exploratória e confirmatória, bem como rigorosos critérios de exclusão de itens, Brown e Ryan (2003) chegaram a uma escala final de natureza unifatorial, composta de 15 itens que refletiam a presença ou a ausência da consciência plena, sendo que os Alfas de Cronbach, nos diferentes estudos, variaram de 0,80 a 0,87 . Estudos posteriores, realizados em amostras norte-americanas (Brown, West, Loverich, \& Biegel, 2011), francesas (Jerman et. al., 2009), espanholas (Galiana, Oliver, Sansó, Sancerni, \& Tomás, 2017), argentinas (Montes, Ledesma, García, \& Poó, 2014), brasileiras (Barros, Kozasa, Souza, \& Ronzani, 2015) e holandesas (Jensen, Niclasen, Vangkilde, \& Petersen, 2016) confirmaram a estrutura unifatorial do instrumento e também apresentaram bons índices de consistência interna.

\section{A consciência plena no trabalho, sua mensuração e relação com variáveis externas}

Ao ser estudada no âmbito do trabalho, a consciência plena pode ser analisada tanto no nível organizacional quanto no individual (Vogus, 2012). No nível organizacional, ela diz respeito ao fato de que as organizações plenamente conscientes possuem a capacidade coletiva de detectar e corrigir erros e eventos inesperados (Vogus, 2012), o que as leva a apresentar um desempenho positivo e livre de erros, mesmo atuando em cenários complexos, interdependentes, dinâmicos e sob pressão temporal, nos quais os erros costumam ser maiores.

No nível individual, a consciência plena no trabalho pode ser conceituada como uma qualidade de atenção elevada dirigida ao ambiente corporativo (Kopelman et. al., 2012), isto é, como o grau em que o trabalhador mostra-se plenamente consciente do que ocorre em seu ambiente laboral (Dane \& Brummel, 2013). Os indivíduos que demonstram consciência plena em seu contexto laboral costumam mostrar-se mais capazes de reduzir seu ritmo interno e observar mais detidamente a ampla gama de experiências que vivenciam, na forma em que elas realmente são, ou seja, em sua totalidade (Marianetti \& Passmore, 2010).

Para avaliar a consciência plena no trabalho, no nível individual, Dane e Brummel (2013) adaptaram para o contexto laboral a escala MAAS (Brown \& Ryan, 2003), tendo dela retirado oito itens, em função de que eles não atendiam ao contexto específico de sua pesquisa, isto é, o trabalho em restaurantes. Os autores não chegaram, porém, a verificar novamente a estrutura interna da escala, nem tampouco sua validade com relação a outras variáveis externas. Um dos objetivos do presente trabalho foi, portanto, avaliar a estrutura interna da ECPT e sua consistência interna em amostras brasileiras. Considerando os estudos de Brown e Ryan (2003) e Dane e Brummel (2013), hipotetizou-se que, na adaptação brasileira da escala, o modelo unifatorial seria o que apresentaria melhor ajuste aos dados (H1).

Adicionalmente, pretendeu-se também reunir evidências de validade convergente do instrumento, mediante a análise das correlações da consciência plena no trabalho com construtos a ela correlatos. Os estudos sobre a consciência plena no contexto do trabalho ainda são escassos, mas têm revelado a existência de relações positivas de tal construto com o desempenho laboral, com o engajamento no trabalho (Dane \& Brummel, 2013) e com a satisfação no trabalho (Hülsheger et al., 2013), bem como negativas, com a intenção de rotatividade (Dane \& Brummel, 2013) e a exaustão emocional (Hülsheger et al., 2013). Tomando por base tais estudos e o fato de que o engajamento no trabalho diz respeito a um estado mental positivo manifesto em sentimentos de vigor, dedicação e absorção ao trabalho (Schaufeli, Bakker, \& Salanova, 2006)), hipotetizou-se que haveria uma correlação positiva moderada ou forte entre a consciência plena no trabalho e o engajamento no trabalho $(\mathrm{H} 2)$.

Outras pesquisas mostraram haver uma associação negativa da consciência plena com indicadores de prejuízo à saúde mental, como a ansiedade (Brown \& Ryan, 2003), a depressão (Klainin-Yobas, et al., 2012) e a exaustão emocional (Hülsheger et al., 2013). Fundamentando-se, portanto, nesses achados e considerando-se que a saúde mental geral diz respeito à ausência de doenças psiquiátricas não severas (Goldberg, 1972), hipotetizou-se que a consciência plena no trabalho apresentaria uma correlação positiva moderada ou forte com a saúde mental geral (H3).

A consciência plena tem também apresentado correlações positivas com a qualidade de vida (Nyklíček, \& Kuijpers, 2008), o bem-estar psicológico (Brown \& Ryan, 2003) e os afetos positivos (Brown \& Ryan, 2003), que dizem respeito a experiências agradáveis de humor e de emoção dirigidas ao trabalho (van Horn, Taris, Schaufeli, \& Schreurs, 2004). Tomando por base tais pesquisas, hipotetizouse que existiria uma correlação positiva moderada ou forte entre a consciência plena no trabalho e os afetos positivos dirigidos ao trabalho (H4).

Investigações anteriores (Brown \& Ryan, 2003) também verificaram a existência de correlações negativas entre a consciência plena e o neuroticismo, que pode ser caracterizado como uma propensão ao desajuste emocional, à instabilidade e a diversos transtornos psicológicos (Nunes \& Hutz, 2002). Assim, hipotetizou-se que haveria uma correlação negativa 
moderada ou forte entre a consciência plena no trabalho e o neuroticismo (H5).

\section{Método}

\section{Participantes}

Foi utilizado um processo de amostragem por conveniência, tendo-se considerado como único critério de inclusão o fato de as pessoas estagiarem ou trabalharem há pelo menos um ano em qualquer tipo de organização. A amostra foi composta por 383 trabalhadores brasileiros de ambos os sexos (65,7\% do sexo feminino), com idades variando entre 18 e 70 anos $(M=34,5 ; D P=11,7)$, sendo que $94,7 \%$ dos trabalhadores apresentaram escolaridade de nível superior incompleto ou completo. Os trabalhadores que responderam à pesquisa provinham de organizações públicas $(36,7 \%)$ e privadas $(63,3 \%)$ e desempenhavam diferentes funções, com 38,8\% trabalhando no nível administrativo ou operacional. O tempo total de serviço variou de 1 a 50 anos $(M=13,2$; $D P=11,4)$.

\section{Instrumentos}

Para a avaliação da consciência plena no trabalho foi adotada uma escala que reuniu os sete itens da Escala de Consciência Plena no Trabalho de Dane e Brummel (2013), e mais quatro itens da escala MAAS (Brown \& Ryan, 2003), que não se aplicavam ao contexto específico de restaurantes, mas que, a partir de uma análise de conteúdo realizada por dois psicólogos organizacionais, foram julgados pertinentes ao contexto do trabalho em geral. Logo, a escala inicial possuía 11 itens, que foram respondidos em escalas Likert de seis pontos $(1=$ quase sempre e $6=$ quase nunca). Um exemplo de item é "Eu faço trabalhos ou tarefas automaticamente, sem estar consciente do que estou fazendo". O processo de adaptação da escala para o idioma português do Brasil envolveu a tradução dos itens da língua inglesa para a portuguesa por dois profissionais bilíngues da Psicologia, a retradução para o inglês por um tradutor norte-americano bilíngue e, em seguida, a submissão a um consenso final das duas traduções por outro profissional bilíngue da área de Psicologia. A inteligibilidade dos itens foi avaliada por um pesquisador da área organizacional não envolvido no processo de tradução e retradução da escala.

O engajamento no trabalho foi avaliado pela versão reduzida da Escala de Engajamento no Trabalho (UWES-9), de Schaufeli et al. (2006), adaptada para o contexto brasileiro por Ferreira et al. (2016). Ela é composta por 9 itens (por exemplo: "Em meu trabalho, sinto-me cheio de energia"), que foram respondidos em escalas tipo Likert de cinco pontos, variando de nunca (1) a diariamente (5). A consistência interna da escala, no presente estudo, avaliada pelo alfa de Cronbach, foi igual a 0,92 .

Para avaliação da saúde mental foi utilizada a versão abreviada do Questionário de Saúde Geral de Goldberg (1972), adaptado para o Brasil por Gouveia et al. (2003). Ele é composto por 12 itens (por exemplo: “Tem perdido a confiança em si mesmo?”), que foram respondidos em escalas do tipo Likert de quatro pontos, variando de absolutamente não (1) a muito mais do que de costume (4). A consistência interna da escala, no presente estudo, foi igual a 0,86 .

O neuroticismo foi medido por meio de uma subescala do Inventário dos Cinco Grandes Fatores de Personalidade (IGFP-5), adaptada para o Brasil por Andrade (2008) e composta por seis itens, que foram respondidos em escalas tipo Likert de cinco pontos. Um exemplo de item é: "Fica nervoso facilmente". O índice de precisão dessa escala, no presente estudo, avaliado pelo Alfa de Cronbach, foi igual a 0,83 .

Os afetos positivos dirigidos ao trabalho foram avaliados pela versão reduzida da subescala de Afetos Positivos, pertencente à Escala de Afetos no Trabalho (ESAFE), de autoria de Ferreira, Silva, Fernandes e Almeida (2008). A escala é unifatorial e contém 10 itens, que foram respondidos em escalas de cinco pontos, variando de nunca (1) a sempre (5). O alfa de Cronbach da escala na presente amostra foi igual a 0,93 . O instrumento de coleta de dados contou, ainda, com um questionário sociodemográfico e com o termo de consentimento livre e esclarecido.

\section{Procedimentos de coleta e análise de dados}

Após a aprovação do Comitê de Ética em Pesquisa (CAAE: 54900316.6.0000.5289), o instrumento foi aplicado em versão eletrônica $(57,9 \%)$ e em lápis e papel $(42,1 \%)$, de forma coletiva ou individual. A aplicação coletiva ocorreu principalmente em estudantes universitários, os quais foram contatados em suas respectivas salas de aula e responderam aos instrumentos na forma de lápis e papel. A aplicação individual ocorreu em trabalhadores de organizações públicas ou privadas, contatados por meio da rede de amizades dos pesquisadores, os quais responderam aos instrumentos por meio eletrônico, com a utilização da ferramenta Google Docs. Em ambas as situações, os participantes receberam inicialmente esclarecimentos acerca dos objetivos da pesquisa e o convite para dela participarem. Os que optaram voluntariamente por participar preencheram o termo de consentimento livre e esclarecido e, em seguida, completaram as escalas 
que, ao final, foram imediatamente devolvidas. Foi assegurado o sigilo acerca das informações prestadas a todos os participantes.

As análises fatoriais confirmatórias foram realizadas por meio do software MPlus (versão 7.11), utilizando-se como método de estimação de parâmetros o weighted least squares mean and variance adjusted (WLSMV) e declarando-se as variáveis como categóricas. Os índices de ajuste avaliados e os valores de referência adotados foram: $\chi^{2} / \mathrm{gl}<5$; CFI $>0,95$; TLI $>0,95 ;$ RMSEA $<0,05$ (Byrne, 2012). A invariância dos parâmetros dos itens em relação ao tipo de aplicação (online ou lápis e papel), sexo, setor de trabalho (público ou privado) e tempo de serviço foi testada no contexto da modelagem por equações estruturais. Para tanto, utilizou-se a modelagem multigrupos (para tipo de aplicação, sexo e setor de trabalho) e a modelagem multiple indicators multiple causes (MIMIC), para o tempo de trabalho. Para a modelagem multigrupos, testou-se as invariâncias configural, métrica e escalar, fixando, em diferentes modelos, o número de itens e fatores, cargas fatoriais e thresholds. As diferenças entre os modelos foram testadas por meio do $\triangle \mathrm{CFI}$, conforme indicadas por Cheung e Rensvold (2002). Para a modelagem MIMIC (Muthén, 1988), testou-se, na primeira etapa, um modelo com o efeito direto entre o tempo de trabalho e a variável latente. Na segunda etapa, controlando os efeitos estimados na etapa anterior, testaram-se as relações diretas entre o tempo de trabalho e os itens da escala. Tais efeitos diretos, se estatisticamente significativos, indicariam que o tempo de serviço influencia a parametrização dos itens.

A avaliação da consistência interna foi realizada por meio do coeficiente Alfa de Cronbach e da confiabilidade composta, sendo que a validade convergente da Escala de Consciência Plena no Trabalho, por meio do cálculo de correlações de Pearson. Na interpretação da magnitude dos coeficientes de correlação foram adotados os critérios de Miles e Shevlin (2001), que os classificam de acordo com os seguintes intervalos: 0,10-0,29 (baixa); 0,30-0,49 (moderada) e superior a 0,50 (elevada).

\section{Resultados}

Com o objetivo de avaliar a estrutura da ECPT, por meio de análise fatorial confirmatória, foram inicialmente calculados os índices de ajuste de um modelo unifatorial contendo os 11 itens da escala original, que apresentou os seguintes indicadores de ajuste: $\chi^{2}(44)=135,958 ; \chi^{2} / \mathrm{gl}=3,09$; $\mathrm{RMSEA}=0,074$ (intervalo de 0,06-0,09), CFI $=0,961 ; \mathrm{TLI}=0,951$. Apesar de tais índices poderem ser considerados razoavelmente satisfatórios, verificou-se que quatro itens apresentaram cargas fatoriais menores que 0,50. Tais itens foram, então, retirados e uma nova análise fatorial confirmatória foi realizada com o modelo reespecificado. Justifica-se a exclusão desses itens pela importância de manter uma escala com poucos itens, sem a perda das características psicométricas da escala original. Os índices obtidos nessa solução revelaramse bastante adequados: $\chi^{2}(14)=16,870 ; \chi^{2} / \mathrm{gl}=1,205$; RMSEA $=0,023$ (intervalo de 0,00-0,06), CFI=0,998; $\mathrm{TLI}=0,998$, razão pela qual este foi o modelo retido, o que confirmou a Hipótese 1.

Os parâmetros não padronizados de tal solução, considerando-se um intervalo de confiança de $95 \%$, foram todos significativamente acima de zero, com valores de razão crítica maiores que 1,96, numa indicação de que todos podem ser considerados úteis ao modelo. A Figura 1 apresenta o modelo final da ECPT e as cargas fatoriais padronizadas. Ainda que alguns parâmetros de regressão padronizados (i. e. cargas fatoriais) tenham apresentado valores abaixo de 0,60, a média desses parâmetros foi igual a 0,65.

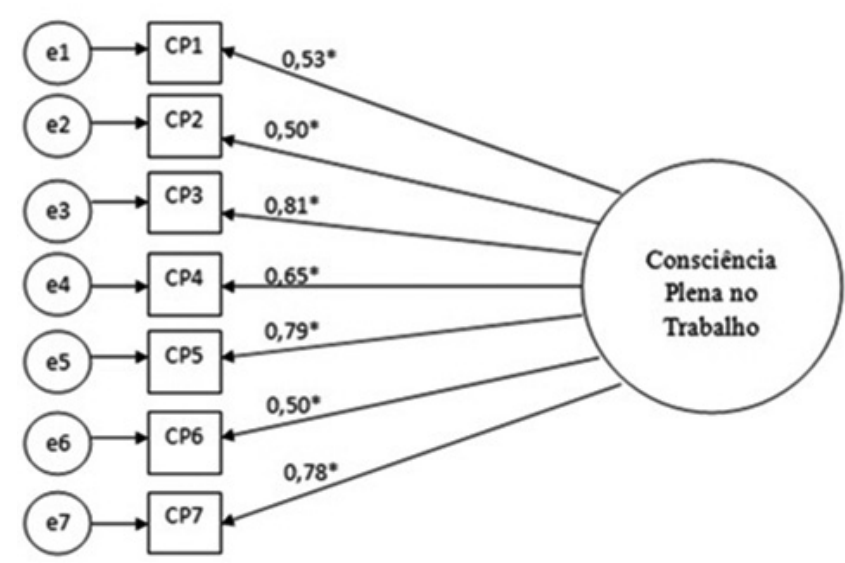

Figura 1. Modelo final da Escala de Consciência Plena no Trabalho com as cargas fatoriais padronizadas.

Com o objetivo de averiguar a invariância dos parâmetros dos itens entre os diferentes grupos relacionados às variáveis tipo de aplicação (online ou lápis e papel), sexo e setor de trabalho (público ou privado) foram realizadas análises fatoriais multigrupos. Os resultados apresentados na Tabela 1 demonstram haver invariância configural (relativa ao número de itens e fatores) e invariância métrica (associada às cargas fatoriais), não tendo sido, porém, encontrada invariância escalar (relacionada aos interceptos) com relação à modalidade de aplicação do instrumento. Uma análise de invariância parcial indicou que, ao menos os thresholds dos itens 2 ("No trabalho, eu acho difícil 
ficar focado no que está acontecendo no presente"), 7 ("No trabalho, eu fico tão focado nos objetivos que quero atingir, que perco o contato com o que estou fazendo agora para chegar lá") e 11 ("No trabalho, eu me pego fazendo coisas sem prestar atenção no que faço") da versão on-line foram mais altos do que os da versão lápis e papel, e na maior parte, essas diferenças foram superiores a 0,50 .

Resultados semelhantes foram encontrados acerca da heterogeneidade dos parâmetros dos itens entre os grupos masculino e feminino. Nesse caso, as dimensões e as cargas fatoriais apresentaram invariância entre os grupos (configural e métrica); no entanto, o modelo com thresholds fixos entre os grupos não se ajustou tão bem aos dados se comparado com os modelos prévios.

TABELA 1

Análise Multigrupo para tipo de aplicação, sexo e setor de trabalho.

\begin{tabular}{|c|c|c|c|c|}
\hline $\begin{array}{c}\text { Modelo e } \\
\text { Variável Externa }\end{array}$ & $\chi^{2}$ & $g l$ & CFI & $\triangle C F I$ \\
\hline \multicolumn{5}{|c|}{$\begin{array}{l}\text { Tipo de aplicação } \\
\text { (online ou lápis e papel) }\end{array}$} \\
\hline Configural & 32,50 & 28 & 0,998 & \\
\hline Métrica & 33,32 & 34 & 1,000 & 0,002 \\
\hline Escalar & 111,20 & 61 & 0,977 & 0,023 \\
\hline Escalar Parcial ${ }^{a}$ & 63,55 & 46 & 0,992 & 0,008 \\
\hline \multicolumn{5}{|l|}{ Sexo } \\
\hline Configural & 38,72 & 28 & 0,994 & \\
\hline Métrica & 45,94 & 34 & 0,994 & $<0,001$ \\
\hline Escalar & 93,78 & 61 & 0,982 & 0,012 \\
\hline Escalar Parcial $^{\text {b }}$ & 74,77 & 57 & 0,990 & 0,004 \\
\hline \multicolumn{5}{|l|}{$\begin{array}{l}\text { Setor de trabalho } \\
\text { (público ou privado) }\end{array}$} \\
\hline Configural & 49,38 & 28 & 0,989 & \\
\hline Métrica & 53,89 & 34 & 0,990 & 0,001 \\
\hline Escalar & 94,07 & 61 & 0,983 & 0,007 \\
\hline
\end{tabular}

${ }^{\mathrm{a}}$ Thresholds livres para os itens 2, 7 e 11; ${ }^{\mathrm{b}}$ Thresholds livres para o item 11.
A análise de invariância parcial indicou, novamente, diferenças entre os thresholds do item 11 ("No trabalho, eu me pego fazendo coisas sem prestar atenção no que faço"). Com relação aos setores público ou privado foi encontrada invariância configural, métrica e escalar.

No que diz respeito ao tempo de serviço, testou-se a invariância da parametrização por meio da modelagem MIMIC (não apresentada nas tabelas). O primeiro modelo, com o controle da relação direta entre a variável externa (observada) tempo de serviço e a variável latente consciência plena se ajustou bem aos dados $\left(\chi^{2}(\mathrm{gl})=16,61(20) ; \mathrm{CFI}=1,00\right)$, e essa relação direta foi fraca e não foi estatisticamente significativa $(\beta=0,05 ; p=0,35)$. No segundo modelo, controlando o efeito direto entre tempo serviço e consciência plena, nenhum item foi explicado, de maneira significativa $(p<0,05)$ diretamente pela variável externa tempo de serviço (ajuste desse modelo: $\chi^{2}(\mathrm{gl})=14,63$ (14); $\mathrm{CFI}=1,00)$. Tais resultados indicam que o tempo de serviço não impacta na parametrização dos itens.

No que se refere à consistência interna, a confiabilidade composta (ou confiabilidade de construto) foi igual a 0,84. Além disso, o Alfa de Cronbach foi igual a 0,81 . Buscou-se, ainda, avaliar as evidências de validade convergente da escala. Para tanto, os escores obtidos em cada um dos instrumentos de coleta de dados foram inicialmente computados e calculadas as médias, desvios-padrões e correlação entre essas escalas (Tabela 2). Os dados obtidos evidenciaram que a Escala de Consciência Plena no Trabalho correlacionou-se de forma positiva e moderada com a Escala de Engajamento no Trabalho $(r=0,32 ; p<0,01)$, com o Questionário de Saúde Geral $(r=0,39 ; p<0,01) \mathrm{e}$ com a Escala de Afetos no Trabalho $(r=0,30 ; p<0,01)$, o que confirmou as Hipóteses 2, 3 e 4, respectivamente. Além disso, ela se correlacionou de forma moderada e negativa com a subescala de neuroticismo do Inventário dos Cinco Grandes Fatores de Personalidade $(r=-0,34$; $p<0,01$ ), o que permitiu também a confirmação da Hipótese 5.

TABELA 2

Médias, desvios-padrões e coeficientes de correlação entre as escalas do estudo

\begin{tabular}{|c|c|c|c|c|c|c|}
\hline Variáveis & $M$ & $D P$ & 1 & 2 & 3 & 4 \\
\hline 1 - Engajamento & 4,02 & 0,81 & - & & & \\
\hline 2 - Saúde mental geral & 2,98 & 0,50 & $0,37 *$ & - & & \\
\hline 3 - Neuroticismo & 2,76 & 0,99 & $-0,22 *$ & $-0,48^{*}$ & - & \\
\hline 4 -Afetos positivos & 3,81 & 0,76 & $0,80^{*}$ & $0,50 *$ & $-0,26^{*}$ & - \\
\hline 5-Consciência plena no trabalho & 4,01 & 1,00 & $0,32 *$ & $0,39 *$ & $-0,34 *$ & 0,30 * \\
\hline
\end{tabular}

$* p<0,01$. 


\section{Discussão}

O presente estudo teve como objetivo investigar as evidências iniciais de validade da Escala de Consciência Plena no Trabalho, em amostras brasileiras. No que diz respeito à análise fatorial confirmatória, os dados obtidos revelaram que o modelo de melhor ajuste foi o modelo reespecificado, em que quatro itens da escala inicial foram retirados, por possuírem cargas fatoriais menores que 0,5 . Dessa maneira, a versão final brasileira da Escala de Consciência Plena no Trabalho manteve quatro itens da escala de Dane e Brummel (2013) e três itens adicionais da escala MAAS (Brown \& Ryan, 2003), ficando assim com sete itens. Os escores dos sete itens conservaram a estrutura unifatorial, de modo semelhante aos modelos originais (Brown \& Ryan, 2003; Dane \& Brummel 2013), o que confirmou a Hipótese 1 da pesquisa. A decisão de retirada desses itens visou permitir que o instrumento ficasse com os melhores índices de ajuste possíveis e, ao mesmo tempo, mantivesse itens suficientes para assegurar uma boa precisão. Além disso, a redução dos itens poderá facilitar coletas posteriores de dados em pesquisas que envolvam vários instrumentos.

Além disso, a escala apresentou um bom índice de confiabilidade composta $(\mathrm{CC}=0,84)$ e Alfa de Cronbach $(\alpha=0,81)$, o que aponta para a possibilidade de estimarem-se os escores latentes de maneira precisa, mesmo por meio da versão reduzida da escala. Tais resultados convergem, portanto, com os obtidos por Dane e Brummel (2013) e por Brown e Ryan (2003) que encontraram, respectivamente, coeficientes alfas iguais a 0,73 e 0,87 , em seus estudos.

As análises de invariância apontaram que a ECPT não apresentou diferenças significativas entre trabalhadores dos setores público ou privado nem tampouco entre trabalhadores com diferentes tempos de serviço. Com relação ao sexo, foi observada invariância configural, métrica e escalar em todos os itens, à exceção do item 11. No que diz respeito às modalidades de aplicação, não foi observada invariância escalar nos itens 2,7 e 11. Tais resultados indicam que as categorias mais altas da escala Likert são mais facilmente endossáveis pelos participantes que responderam à versão lápis e papel do instrumento. Essa sistemática tendência de endosso das categorias mais altas entre os participantes que realizaram a pesquisa presencialmente pode estar associada à desejabilidade social e merece ser objeto de investigações futuras. De modo geral, porém, os achados ora obtidos atestam a equivalência da estrutura da escala em diferentes grupos e formas de aplicação.

No que diz respeito à validade convergente da escala, observou-se que a consciência plena no trabalho correlacionou-se de forma positiva e moderada com o engajamento no trabalho $(r=0,32)$, confirmando assim a Hipótese 2. Tal achado mostra-se consistente com os relatados por Brown e Ryan (2003), que também encontraram uma correlação moderada positiva entre a consciência plena e o engajamento $(r=0,33)$. Nesse sentido, quando o trabalhador direciona totalmente sua consciência para as atividades que lhe compete executar, sem se deixar levar por preocupações e distrações externas ao trabalho, ele costuma se mostrar mais envolvido com suas tarefas e, consequentemente, manifesta mais sentimentos de vigor, dedicação e absorção a tais atividades (Dane \& Brummel, 2013).

Uma correlação positiva e moderada $(r=0,39)$ foi também observada entre a consciência plena no trabalho e a saúde mental, confirmando, assim, a Hipótese 3. Tal achado vai ao encontro dos de Brown e Ryan (2003), que observaram correlações moderadas entre a consciência plena e distúrbios emocionais opostos à saúde mental, como a depressão $(r=-0,41)$ e a ansiedade $(r=-0,40)$. Uma possível explicação para esses resultados é que as pessoas plenamente conscientes do que acontece em seus ambientes de trabalho cuidam mais de sua saúde mental geral, demonstrando, por conseguinte, ausência de doenças psiquiátricas não severas, tais como a depressão e a ansiedade. Em outras palavras, a consciência plena no trabalho favorece o desenvolvimento de atitudes subjetivas mais conscientes frente a experiências emocionalmente aversivas as quais, por sua vez, contribuem para que os indivíduos se tornem mais aptos a lidar com essas experiências, de forma a evitar o surgimento de doenças psiquiátricas não severas e a manter bons níveis de saúde mental (Bishop et al., 2004; Brown \& Ryan, 2003; Piet, Wurtzen, \& Zachariae, 2012).

A correlação da consciência plena no trabalho com os afetos positivos dirigidos ao trabalho $(r=0,30)$ foi moderada e positiva, o que sustentou empiricamente a Hipótese 4. O presente resultado assemelha-se ao verificado por Brown e Ryan (2003), que identificou uma correlação igual à obtida na atual investigação. Tal resultado pode se dever ao fato de que os trabalhadores, em razão de desenvolverem maior consciência do que acontece em seus ambientes laborais, acabam por focalizar sua atenção nos melhores aspectos desse ambiente, o que, por conseguinte, leva-os a vivenciar mais experiências agradáveis de humor e de emoção em seu contexto laboral (Brown \& Ryan, 2003).

No que diz respeito à correlação da consciência plena no trabalho com o neuroticismo, foi verificada uma associação moderada e negativa entre esses construtos $(r=-0,34)$, o que levou à confirmação da 
Hipótese 5. Esse resultado alinha-se com os de Brown e Ryan (2003), que também encontraram uma correlação moderada e negativa $(r=-0,33)$ da consciência plena com o neuroticismo. De acordo com Brown e Ryan (2003), os trabalhadores plenamente conscientes de suas responsabilidades laborais tendem a ser menos neuróticos porque identificam com mais acurácia a oscilação de suas emoções e, consequentemente, adotam mais frequentemente atitudes psicológicas profiláticas que vão na direção do bem-estar, o que os protege dos desajustes emocionais, da instabilidade e da manifestação de transtornos mentais típicos do neuroticismo (Bishop et al., 2004).

Cumpre registrar, porém, como limitação do estudo, o fato de a amostra ter sido composta por participantes, em sua maioria, do estado do Rio de Janeiro, o que dificulta a generalização dos resultados para os demais estados brasileiros. Outra limitação diz respeito à ausência de invariância escalar total entre as amostras de sexo masculino e feminino e as amostras on-line e em lápis e papel, o que não assegura a plena equivalência das estimativas dos escores latentes entre essas amostras, razão pela qual tal equivalência mereceria ser investigada em maior profundidade em pesquisas posteriores.

Considerando-se, ainda, que $94,7 \%$ da amostra possuía nível superior (completo ou incompleto), é possível também que tal variável possa ter exercido alguma influencia nos atuais resultados. Assim, recomenda-se o desenvolvimento de estudos futuros com amostras de outros níveis de escolaridade.
Vale mencionar, por fim, que o instrumento original utilizado na presente adaptação (Dane \& Brummel, 2013) apresenta uma escala de respostas aos itens que varia de 1 (quase sempre) a 6 (quase nunca). Tal ancoragem, por trazer uma escala invertida, pode acabar por confundir o respondente. Contudo, na presente investigação, decidiu-se por manter a fidelidade ao instrumento original e não modificar a posição das âncoras. Seria interessante, porém, que estudos futuros verificassem a existência ou não de diferenças entre ambas as modalidades de resposta ao instrumento.

Ainda no que tange à realização de estudos futuros, sugere-se que sejam realizadas pesquisas que objetivem o aprofundamento da rede nomológica do construto consciência plena no trabalho, em especial no que diz respeito a seu impacto em variáveis que caracterizam o bem-estar e a qualidade de vida no trabalho e fora dele, como por exemplo, o comportamento de redesenho no trabalho, o enriquecimento trabalho-família e a cidadania organizacional. Tais pesquisas poderiam ser de natureza longitudinal, o que permitiria maior compreensão das relações entre essas variáveis.

De todo modo, os resultados do presente estudo indicam que a Escala de Consciência Plena no Trabalho apresentou evidências iniciais de validade de estrutura interna, precisão e validade convergente, em uma amostra brasileira. Portanto, ela pode ser considerada como uma medida apropriada à avaliação do quão plenamente conscientes as pessoas se mostram em seus ambientes laborais, o que recomenda seu uso futuro em pesquisas e em diagnósticos organizacionais.

\section{Referências}

Andrade, J. M. (2008). Evidencias de validade do inventário dos cinco grandes fatores de personalidade para o Brasil. Tese de doutorado não publicada em Psicologia Social, do Trabalho e das Organizações. Universidade de Brasília, UnB.

Barros, V. V., Kozasa, E. H., Souza, I. C. W., \& Ronzani, T. M. (2015). Validity evidence of the Brazilian version of the Mindful Attention Awareness Scale (MAAS). Psicologia: Reflexão e Crítica, 28, 87-95. https://doi.org/10.1590/16787153.201528110

Bishop, S. R., Lau, M., Shapiro, S., Carlson, L., Anderson, N. D, Carmody, J., Segal, Z. V., Abbey, S., Speca, M., Velting, D., \& Devins, G. (2004). Mindfulness: A proposed operational definition. Clinical Psychology: Science and Practice, 11, 230-241. https://doi.org/10.1093/clipsy.bph077

Brown, K. W. \& Ryan, R. M. (2003). The benefits of being present: Mindfulness and its role in psychological well-being. Journal of Personality and Social Psychology, 84, 822-848. https://doi.org/10.1037/0022-3514.84.4.822

Brown, K. W., West, A. M., Loverich, T. M., \& Biegel, G. M. (2011). Assessing adolescent mindfulness: Validation of an adapted Mindful Attention Awareness Scale in adolescent normative and psychiatric populations. Psychological Assessment, 23, 1023-1033. https://doi.org/10.1037/a0021338

Byrne, B. M. (2012). Structural equation modeling with Mplus: Basic concepts, applications, and programming. New York: Taylor \& Francis.

Cheung, G. W., \& Rensvold, R. B. (2002). Evaluating goodness-of-fit for testing measurement invariance. Structural Equation Modeling, 9, 233-255. https://doi.org/10.1207/S15328007SEM0902_5

Dane, E. \& Brummel, B. J. (2013). Examining workplace mindfulness and its relations to job performance and turnover intention. Human Relations, 67, 105-128. https://doi.org/10.1177/0018726713487753 
Ferreira, M. C., Silva, A. P. C., Fernandes, H., \& Almeida, S. P. (2008). Desenvolvimento e validação de uma escala de afetos no trabalho (ESAFE). Revista de Avaliação Psicológica, 7, 143-150.

Ferreira, M. C., Valentini, F., Damásio, B. F., Mourão, L., Porto, J., Chinelato, R. S. C., Novaes, V. P., \& Pereira, M. M. (2016). Evidencias adicionais de validade da UWES-9 em amostras brasileiras. Estudos de Psicologia, prelo. https:// doi.org/10.5935/1678-4669.20160042

Galiana, L., Oliver, A., Sansó, N., Sancerni, M. D., \& Tomás, J. M. (2017). Mindful Attention Awareness in Spanish palliative care professionals: Psychometric study with IRT and CFA models. European Journal of Psychological Assessment, 33, 14-21. https://doi.org/10.1027/1015-5759/a000265

Goldberg, D. (1972). The detection of psychiatric illness by questionnaire. London: Oxford University Press. https://doi. org/10.1017/S0033291700048613

Gouveia, V., Chaves, S. S. S., Oliveira, I. C. P., Dias, M. R., Gouveia, R. S. V., \& Andrade, P. R. (2003). A utilização do QSG-12 na população geral: Estudo de sua validade de construto. Psicologia: Teoria e Pesquisa, 19(3), 241-248. https://doi.org/10.1590/S0102-37722003000300006

Houben, M., van den Noortgate, W., \& Kuppens, P. (2015). The relation between short-term emotion dynamics and psychological well-being: A meta-analysis. Psychologica Bulletin, 141, 901-930. https://doi.org/10.1037/ a0038822

Hülsheger, U. R., Alberts, H. J. E. M., Feinholdt, A., \& Lang, J. W. B (2013). Benefits of mindfulness at work: The role of mindfulness in emotion regulation, emotional exhaustion, and job satisfaction. Journal of Applied Psychology, 98, 310-325. https://doi.org/10.1037/a0031313

Jensen, C. G., Niclasen, J., Vangkilde, S. A., \& Petersen, A. (2016). General inattentiveness is a long-term reliable trait independently predictive of psychological health: Danish validation studies of the Mindful Attention Awareness Scale. Psychological Assessment, 28, 70-87. https://doi.org/10.1037/pas0000196.supp

Jermann, F., Billieux, J., Laroi, F., d'Argembeau, A., Bondolfi, G., Zermatten, A., \& Van der Linden, M. (2009). Mindful Attention Awareness Scale (MAAS): Psychometric properties of the French translation and exploration of its relations with emotion regulation strategies. Psychological Assessment, 21, 506-514. https://doi.org/10.1037/ a0017032

Klainin-Yobas, P., Cho, M. A. A., \& Creedy, D. (2012). Efficacy of mindfulness-based interventions on depressive symptoms among people with mental disorders: A meta-analysis. International Journal of Nursing Studies, 49, 109-121. https://doi.org/10.1016/j.ijnurstu.2011.08.014

Kopelman, S., Avi-Yonah, O., \& Varghese, A. K. (2012). The mindful negotiator: Strategic emotion management and well-being. In K. S. Cameron, \& G. M. Spreitzer (Eds.). The Oxford handbook of positive organizational scholarship (1 ${ }^{\text {st }}$ ed., pp. 591-600). Oxford: Oxford University Press. https://doi.org/10.2139/ssrn.1866504

Marianetti, O. \& Passmore, J. (2010). Mindfulness at work: Paying attention to enhance well-being and performance. In P. A. Linley, S. Harrington, \& N. Garcea (Eds.). Oxford handbook of positive psychology and work ( $1^{\text {st }}$ ed.) (pp. 189-200). Oxford: Oxford University Press. https://doi.org/10.1093/oxfordhb/ 9780195335446.013. 0015

Miles, J. N. V. \& Shevlin, M. E. (2001). Applying regression and correlates: A guide for students and researchers. London: Sage Publications.

Montes, S. A., Ledesma, R. D., García, N. M., \& Poó, F. M. (2014). The Mindful Attention Awareness Scale (MAAS) in an Argentine population. Measurement and Evaluation in Counseling and Development, 47, 43-51. https://doi. org/10.1177/0748175613513806

Muthén, B. (1988). Some uses of structural equation modeling in validity studies: Extending IRT to external variables. In H. Wainer, \& H. Braun (Eds.). Test validity (pp. 213-238). Hillsdale, NJ: Erlbaum.

Nunes, C. H. S. S. \& Hutz, C. (2002). O modelo dos Cinco Grande Fatores de Personalidade. In R. Primi (Ed.). Temas em Avaliação Psicológica (pp. 40-49). São Paulo: Casa do Psicólogo.

Nyklíček, I. \& Kuijpers, K. F. (2008). Effects of mindfulness-based stress reduction intervention on psychological well-being and quality of life: Is increased mindfulness indeed the mechanism? Annals of Behavioral Medicine, 35, 331-340. https://doi.org/10.1007/s12160-008-9030-2

Piet, J., Würtzen, H., \& Zachariae, R. (2012). The effect of mindfulness-based therapy on symptoms of anxiety and depression in adult cancer patients and survivors: A systematic review and meta-analysis. Journal of Consulting and Clinical Psychology, 80, 1007-1020. https://doi.org/10.1037/a0028329

Schaufeli, W. B., Bakker, A. B., \& Salanova, M. (2006). The measurement of work engagement with a short questionnaire: A cross-national study. Educational and Psychological Measurement, 66, 701-716. https://doi. org/10.1177/0013164405282471

van Horn, J. E., Taris, T. W., Schaufeli, W. B., \& Schreurs, P. J. G. (2004). The structure of occupational well-being: A study among Dutch teachers. Journal of Occupational and Organizational Psychology, 77, 365-375. https://doi. org/10.1348/0963179041752718

Vogus, T. J. (2012). Mindful organizing: Establishing and extending the foundations of highly reliable performance. In K. S. Cameron \& G. M. Spreitzer (Eds.). The Oxford handbook of positive organizational scholarship (1 ${ }^{\text {st }}$ ed.) (pp. 591-600). Oxford: Oxford University Press. https://doi.org/10.1093/oxfordhb/ 9780199734610.013.0050 
Winbush, N. Y., Gross, C. R., \& Kreitzer, M. J. (2007). The effects of mindfulness-based stress reduction on sleep disturbance: A systematic review. Explore: Journal of Science and Healing, 3, 585-591. https://doi.org/10.1016/j. explore.2007.08.003

Wolever, R. Q., Bobinet, K. J., McCabe, K., Mackenzie, E. R., Fekete, E., Kusnick, C. A., \& Baime, M. (2012). Effective and viable mind-body stress reduction in the workplace: A randomized controlled trial. Journal of Occupational Health Psychology, 17, 246-258. https://doi.org/10.1037/a0027278

\section{Autores:}

André Luis Amorim Silva Filho - Mestre, Universidade Salgado de Oliveira.

Maria Cristina Ferreria - Doutora, Universidade Salgado de Oliveira.

Felipe Valentini - Doutor, Universidade Salgado de Oliveira.

\section{Endereço para correspondência:}

André Luis Amorim Silva Filho

Rua Sá Viana, 107 apto. 403

20540-260 - Grajaú, RJ, Brasil

<aamorimsilvafilho@yahoo.com.br>

Recebido em: 18.05.2016

Aceito em: 28.03.2017 